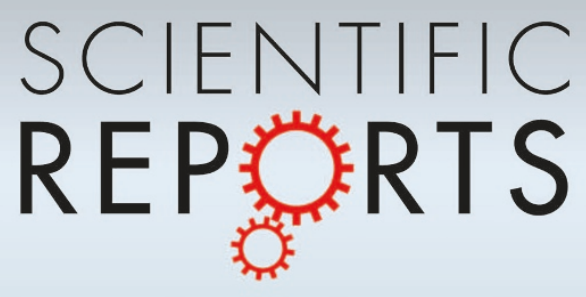



SUBJECT AREAS:

NANOFABRICATION AND NANOPATTERNING

NANOSTRUCTURES

INTERFERENCE MICROSCOPY

MOLECULAR MACHINES AND MOTORS

Received

1 November 2012

Accepted

21 December 2012

Published

21 January 2013

Correspondence and requests for materials should be addressed to M.L. (mercy.lard@ftf. Ith.se) or H.L. (heiner. linke@fff.Ith.se)

\section{Tracking Actomyosin at Fluorescence Check Points}

\author{
Mercy Lard', Lasse ten Siethoff ${ }^{2}$, Alf Månsson ${ }^{2}$ \& Heiner Linke'
}

1The Nanometer Structure Consortium (nmC@LU) and Division of Solid State Physics, Lund University, Box 118, SE-221 00 Lund,
Sweden, ${ }^{2}$ School of Natural Sciences, Linnaeus University, SE-391 82 Kalmar, Sweden.

Emerging concepts for on-chip biotechnologies aim to replace microfluidic flow by active, molecular-motor driven transport of cytoskeletal filaments, including applications in bio-simulation, biocomputation, diagnostics, and drug screening. Many of these applications require reliable detection, with minimal data acquisition, of filaments at many, local checkpoints in a device consisting of a potentially complex network of channels that guide filament motion. Here we develop such a detection system using actomyosin motility. Detection points consist of pairs of gold lines running perpendicular to nanochannels that guide motion of fluorescent actin filaments. Fluorescence interference contrast (FLIC) is used to locally enhance the signal at the gold lines. A cross-correlation method is used to suppress errors, allowing reliable detection of single or multiple filaments. Optimal device design parameters are discussed. The results open for automatic read-out of filament count and velocity in high-throughput motility assays, helping establish the viability of active, motor-driven on-chip applications.

n living cells, some molecular motors walk along cytoskeletal filaments such as actin filaments or microtubules. In motility assays based on the actomyosin ${ }^{1,2}$ or kinesin-microtubule ${ }^{3}$ motor systems, surfaceadsorbed motors power filament motion. By adsorbing the motors to nanoscale tracks for high-precision guiding of filaments ${ }^{4-8}$ and by attaching cargoes $^{9-11}$ this motion can substitute the bulky pumps typically required for microfluidic devices ${ }^{12-14}$. Specifically, much effort has recently been expended to develop nanotechnological applications $^{8,15-20}$ e.g. in molecular sorting ${ }^{21}$, biosensing, diagnostics ${ }^{20,22}$, biocomputation ${ }^{23-27}$ and drug discovery ${ }^{8}$.

In many of the above applications it is essential to detect, with low error rate, the passing of a given filament at certain positions along a track. This can be required to enable steering into a certain direction at a junction (for example in molecular sorting) or to detect changes in velocity (for example in drug-discovery or diagnostic devices). A particularly demanding and potentially high-impact application of the detection of motor-driven filaments is in biocomputation ${ }^{24,25}$. Here, a combinatorial problem (such as the subset-sum problem ${ }^{23}$ ) would be encoded into a physical channel network including switches or nodes. Using many motile agents that explore all possible pathways present in this network (for simplicity represented as a black box in Fig. 1), a large problem can be solved in a highly parallel manner, and potentially faster than in traditional computers ${ }^{24,25}$. A solution is represented by the subset of exits from which motile agents emerge (Fig. 1). Thus, the number of filaments emerging from a potentially very large number of exits must be counted efficiently and accurately.

In recent proof-of-principle experiments the motion of motor-driven filaments has usually been tracked stepby-step using image processing ${ }^{21,28-31}$. However, in order to take full advantage of the scalability of parallel, motordriven devices in applications as described above, it is necessary to consider potentially very large and multiplexed networks. In such up-scaled devices it will not be feasible to globally record the motion of all filaments with sufficient resolution and signal-to-noise $(\mathrm{S} / \mathrm{N})$ by using standard epifluorescence techniques, and it will computationally not be feasible to track each filament. Instead, a detection system is needed that (i) can monitor a potentially very large number of positions along the nanoscale tracks, (ii) requires a minimum number of sensing channels at each position and (iii) uses $\mathrm{S} / \mathrm{N}$ enhancement and error correction to reliably detect all filaments.

Here, we develop the key elements of such a local, data-efficient detection system (Fig. 1(a)). Detection points consist of Au lines running perpendicular to a nanofabricated filament-guiding channel. Fluorescence-interference contrast (FLIC, see below) is used to enhance the signal from fluorescently labelled filaments moving across a $\mathrm{Au}$ line. This $\mathrm{S} / \mathrm{N}$ enhancement is additionally improved, by more than one order of magnitude, by crosscorrelating the signals from a pair of detectors. The sensing requirements are minimal, requiring the read-out of only one or a few pixels of a CCD device per Au line.

As our molecular-motor system of choice, we use actin filaments powered by myosin attached to the device surface (Fig. 1(b) $)^{8}$. The actomyosin system has the advantages of high speed (up to $10 \mu \mathrm{m} / \mathrm{s}$, allowing high 


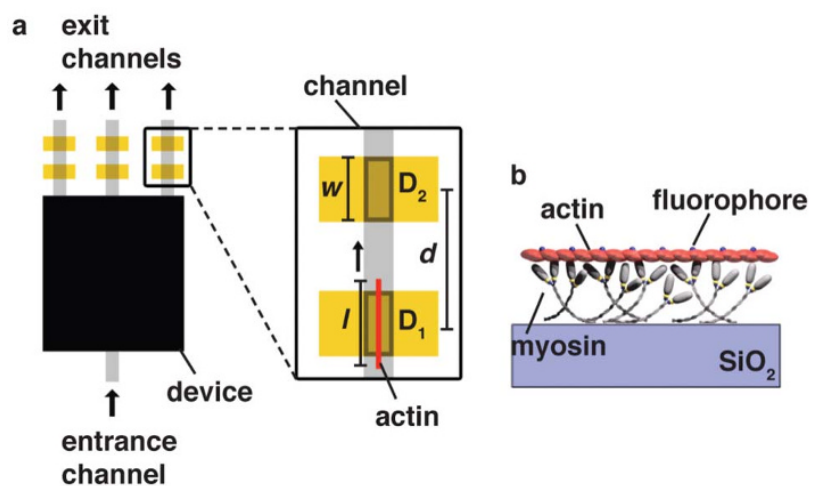

Figure $1 \mid$ Schematic view of proposed readout regions in a future device, such as a biocomputation device. a) The device itself is schematically shown as a black box, with entrance and exit channels. Detector points are here indicated at each exit channel of the network (but could be placed at any point of the device, as required by the application). Each detector point consists of a Au line of width $w$, for FLIC enhancement. The fluorescence originating from actin filaments $l$, crossing detection regions $D_{1}$ and $D_{2}$ from a pair of $\mathrm{Au}$ lines, with centre-to-centre spacing $d$, is read out by one or more CCD pixels each, and cross correlation analysis is used to remove errors from each of the signals. b) Schematic of actin transport by myosin on a $\mathrm{SiO}_{2}$ surface in an in vitro motility assay.

throughput in future devices) and high flexibility, enabling improved miniaturization of channel networks. In the following we describe the electron-beam lithography (EBL) based fabrication process of the nanochannels and detector Au lines, including the novel use of a hydrogen silsesquioxane (HSQ) layer on top of the Au to support actin motility. We further demonstrate that cross-correlation of the FLIC signals from pairs of Au lines can be used to strongly reduce error rates, and discuss the ideal width and spacing of the Au lines for the detection of individual filaments and for the resolution of filaments moving close to one another.

\section{Results}

The overall device layout is shown in Figure 2: it consists of nanochannels (Fig. 2(a)), with a slight undercut for better filament confinement (Fig. 2(b) $)^{4}$, that lead across a series of Au lines used for FLIC enhancement. Large open areas, so called loading zones (Fig. 2(c)), are used to locally bind myosin and actin, with high density, to the surface. Filaments move across the myosin-coated surface, and are guided to the channels by the funnel shape of the loading zones ${ }^{4}$. See Methods for fabrication details.

Fluorescence interference contrast. FLIC creates contrast by destructive and/or constructive interference of light reflected off the substrate surface. Here we make use of FLIC to locally enhance the fluorescence signal of the filaments passing over Au lines (see Fig. 3 and Methods for more information). In Figure 4 we show data of the fluorescence intensity observed, as a function of position, in a detection area as a filament moves across a pair of Au lines. Firstly, the data establish actomyosin motility on top of trimethylchlorosilane (TMCS)-derivatized SiOx deposited by HSQ. Second, a clear peak of the optical intensity was observed when an actin filament was on top of the Au line thus establishing the potential of the FLIC-based detection of actin filaments.

Cross correlation. In an actual device with many filament-detection points, it is desirable to optically read out only a small region (such as $D_{1}$, see Fig. 1(a)), in order to minimize the amount of data to be processed, and to increase achievable feature density. The black line in Figure 5(a) shows the background-subtracted fluorescence signal $D_{1}(t)$ measured in area $D_{1}$ as a function of time as a number of filaments pass through the detection region. Filaments can then be counted directly from the number of detection peaks, but at the risk of introducing errors: free filaments floating by in solution can give false positives, and short or weakly fluorescing filaments may be difficult to distinguish from background noise. Also, a short dip in fluorescence between two closely spaced filaments can be difficult to distinguish from noise-related features. We therefore used a



Figure $2 \mid$ Nanochannels for filament guiding with Au lines for FLIC detection. a) Cross-section of nanochannel with Au line. Approximate thicknesses of resist layers: polymethyl methacrylate (PMMA) and lift-off resist (LOR), and widths of channels similar to (b) are indicated. b) Scanning electron micrograph of nanochannel profile. Coating with $10 \mathrm{~nm}$ Pt for SEM viewing. c) Bright field image of overview of one device with two loading zones connected by two parallel nanochannels. Au lines $(w=2 \mu \mathrm{m}$, see Fig. 1) run perpendicular to channels, with Au-line centre-to-centre spacing of $d=5 \mu \mathrm{m}$. 

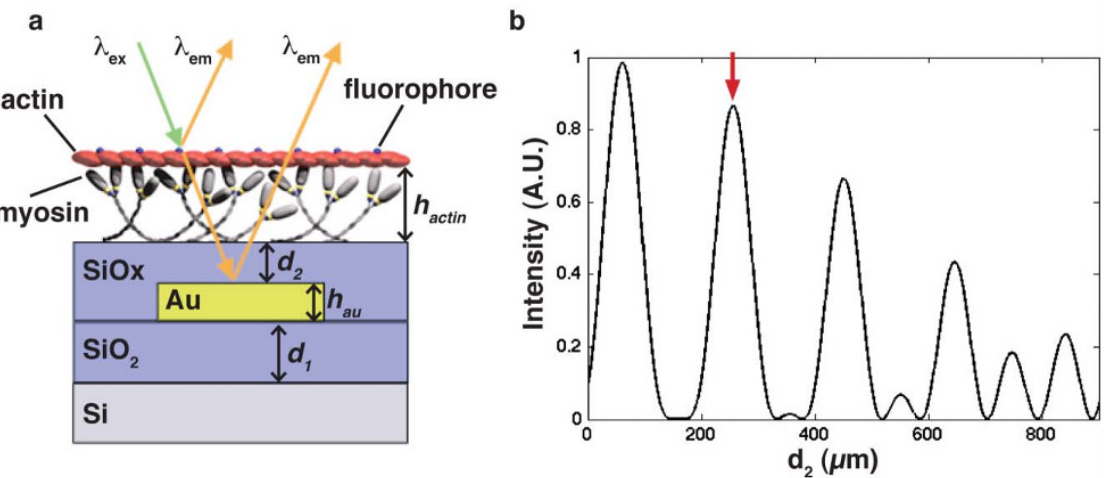

Figure 3 Schematic of FLIC concept and response curve for enhancement of filament contrast. a) Myosin on the SiOx surface transports fluorescently labelled actin filaments at a height, $h_{\text {actin }}$, above the SiOx surface. Incoming light excites the labelled actin filament causing fluorescence emission. Emitted light $\left(\lambda_{\mathrm{em}}\right)$ can travel directly to the detector or indirectly via reflection from the Au surface. Enhancement of fluorescence results if the two emission signals are in phase. The combination of reflective surfaces can be used to manipulate the fluorescence signal both on and off the Au lines. $b$ ) Varying the thickness, $d_{2}$, shows the theoretical maximum and minimum intensities due to FLIC, see equation (2). Excitation $\lambda_{\mathrm{ex}}$ and emission $\lambda_{\mathrm{em}}$ wavelengths are 540 and $605 \mathrm{~nm}$ for Tetra methyl Rhodamine Iso-Thiocyanate (TRITC) filter. Red arrow indicates corresponding thickness used for enhanced TRITC fluorescence on top of Au (see Fig. 4).

cross-correlation analysis technique to strongly enhance the $\mathrm{S} / \mathrm{N}$ ratio. We achieved this by including two Au lines, separated by $5 \mu \mathrm{m}$ (inset to Fig. 5(b)) as individual detector regions, and obtained fluorescence signals, $D_{1}(t)$ and $D_{2}(t)$, from each detector $\left(D_{1}\right.$ and $D_{2}$ in the inset to Fig. 5(b)), shown as the black and the brown line, respectively in Figure 5(b).

Clearly, the main features in the fluorescence profiles were similar but separated in time by some delay. Noise, on the other hand, is not correlated between the two signals and can thus be eliminated as described in the following steps.

Background subtraction is first done to reduce effects from bleaching. Here we apply a simple linear fit to the data to find a background signal, which is then subtracted from the data. Next, the cross correlation of the two fluorescence profiles can be described by:

$$
I_{\text {corr }}=\sum_{0}^{t_{\text {end }}} D_{1}[t] D_{2}\left[t+t_{s}\right],
$$

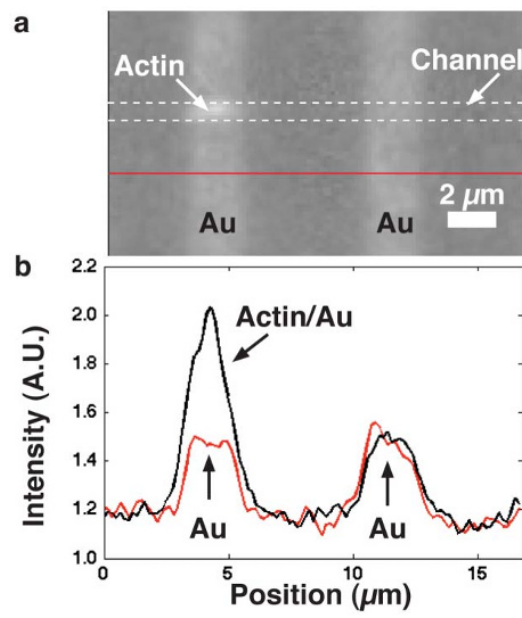

Figure $4 \mid$ Enhanced fluorescence contrast of actin filament in nanochannel. a) An actin filament labelled with $\mathrm{RhPh}$ was imaged using a TRITC filter. The approximately $l=2.5 \mu \mathrm{m}$ long filament passed over the $w=2 \mu \mathrm{m}$ wide Au lines, with $d_{2}=240 \mathrm{~nm}$ (see Fig. 3 ), and there became brighter. b) fluorescence profile along the actin-guiding channel (dashed white lines in (a)) and below channel region (red line in (a)). A single pixel line along the centre of the channel was used to create the profiles shown. where $t_{\mathrm{s}}$ is the total shift used, and $t_{\text {end }}$ is the total time recorded minus $t_{\mathrm{s}}$. We shift the signal $D_{2}(t)$ back in time in $0.2 \mathrm{~s}$ increments with respect to $D_{1}(t)$. The resulting $I_{\text {corr }}$ as a function of $t_{\mathrm{s}}$ is shown in Figure 5(c). The bin size in Figure 5(c) is here set by the frame rate and corresponds also to the expected standard deviation $(0.16 \mathrm{~s})$ of filament travel times between the Au lines, resulting from fluctuations in filament velocity (see below). For a time delay of $t_{\text {smax }} \approx 0.8 \mathrm{~s}$ (the location of the peak in Fig. 5(c)) the signals originating from the two detectors for the same filament are correlated. This time delay is consistent with $\Delta t=d / v \approx 0.9 \mathrm{~s}$, based on the measured speed of the filaments $v \approx 8 \pm 1.4 \mu \mathrm{m} / \mathrm{s}$ (mean $\pm \mathrm{SD}, \mathrm{n}=10$ ), and the centre-tocentre Au-line spacing of $d=7 \mu \mathrm{m}$. The speed is measured from actin filaments transported in the nanochannels on the same surface using a previously described tracking program ${ }^{32}$.

As a next step, the $D_{2}(t)$ signal is shifted by $t_{\text {smax }}=0.8 \mathrm{~s}$ to overlap with the signal $D_{1}(t)$, and the two signals are multiplied. This step effectively suppresses uncorrelated noise, and enhances the correlated, real signal. Finally, the resulting signal is normalized to a maximum peak value of one (Fig. 5(d)).

Now, we wish to automatically extract peaks. We use a simple algorithm that looks for the median value in a window size of $0.4 \mathrm{~s}$ (two frames). This window is shifted in steps of $0.2 \mathrm{~s}$ (single frames) over the entire data set. The median value is compared to a threshold of $T$, chosen here to be $T=0.01$, taken as a fraction of the normalized data set. If the median value is less than $T$, then it is assigned a zero value, if it is higher than or equal to this threshold value, the value at this point is returned (Fig. 5(e), black curve). Finally the peaks are extracted by finding the local maximum over a range of $0.6 \mathrm{~s}$ (three frames; Fig. 5(e), red arrows). The number of peaks extracted in this way gives the number of individual filaments that passed by the detector during the observation. For automated data processing it is important that a range of $T$ values gives reliable results. In Figure 5(f) we show that $0.004<T<0.01$ produces the correct number of filaments, as verified by direct viewing. A $T$ value outside this range produces false positives (for $T<0.004$ ) or misses filaments (for $T>0.01$ ).

For comparison, we use the same peak finding method as in Figure $5(\mathrm{e})$ but applied to data $\left(D_{1}(t)\right)$ from only one detector. The result is shown by red arrows in Figure 5(a). Clearly, a large number of false positives is produced, which are effectively eliminated by the above cross-correlation method. Whereas more advanced peak finding algorithms exist ${ }^{33-35}$, for transparency, we here used a simple method to illustrate the process. Our results demonstrate that the correlation technique effectively suppresses false positives, which will 

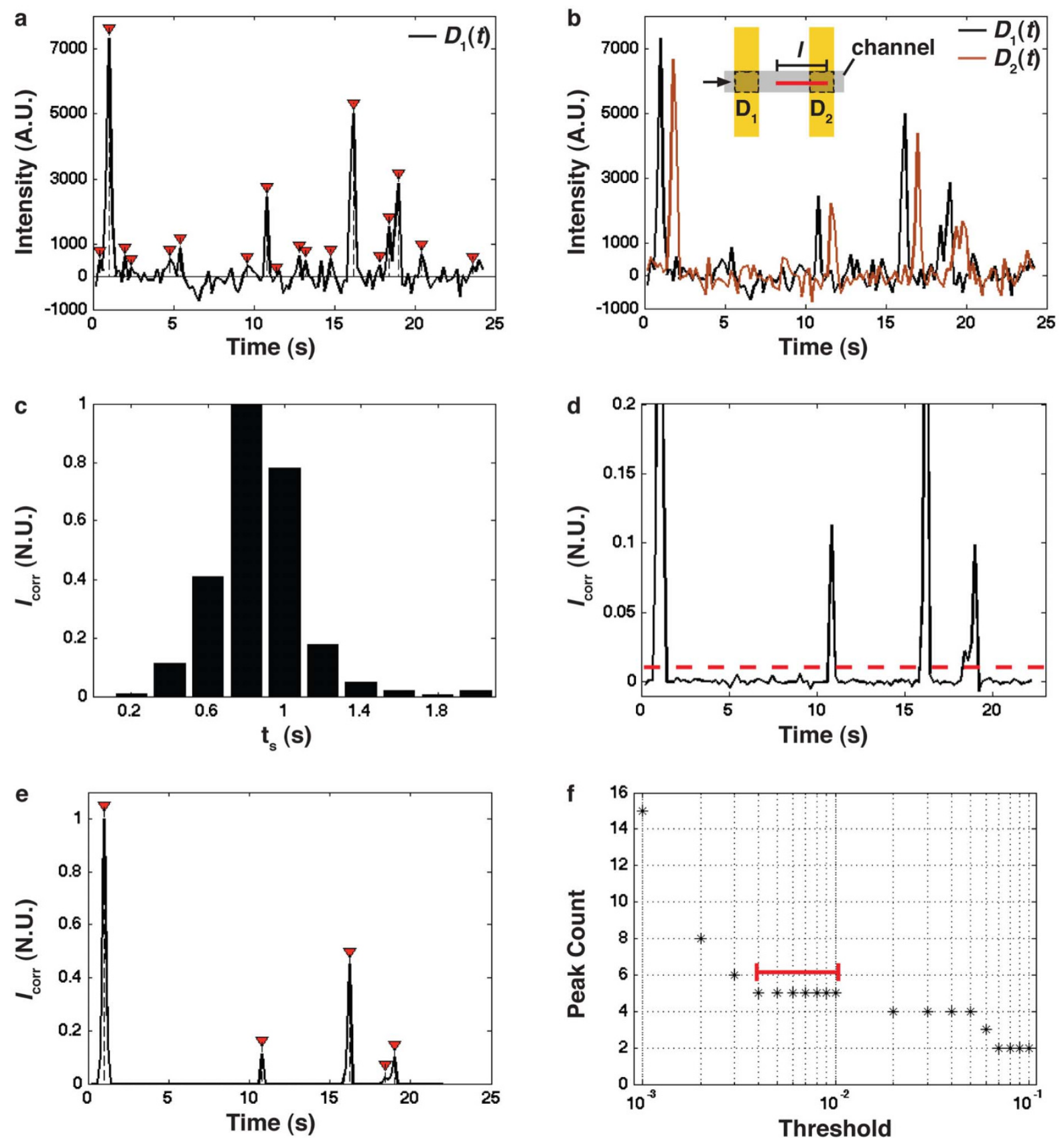

Figure $5 \mid$ Cross correlation of FLIC signals for single filament detection. a) Time series trace of fluorescence recorded in detector region $\mathrm{D}_{1}$ as filaments move over the Au line. Red arrows: peaks identified using the same peak-finding algorithm as in (e), resulting in many false positives: according to visual inspection, five filaments passed by the detector. A constant background was subtracted from the data. b) Background-subtracted fluorescence recorded in detector regions $\mathrm{D}_{1}$ and $\mathrm{D}_{2}$, each consisting of one Au line $(w=2 \mu \mathrm{m})$, with $d=7 \mu \mathrm{m}$ (see Fig. 1$)$. Inset: Schematic of detector regions. c) Cross correlation signal $I_{\text {corr }}$ (see equation (1)) of the $D_{1}(t)$ and $D_{2}(t)$ signals from (b), as a function of $t_{\mathrm{s}}$, indicating that $I_{\text {corr }}$ is maximal for $t_{\mathrm{s}, \max }=0.8 \mathrm{~s}$. d) The multiplied signal $D_{1}(t)^{*} D_{2}\left(t+t_{\mathrm{s}, \max }\right)$ after normalizing. The dashed line shows the threshold level $T=0.01 \mathrm{used}$ to produce the signal in (e). e) Black curve: Same data as in (d) after subtraction of 0.01 of the maximum. Red arrows: extracted peaks using a peak-finding algorithm (see text). f) Number of peaks identified as a function of the threshold level $T$ applied to the cross correlated data set from (d), spanning from 0.001-0.1 of the maximum. Red bar: a threshold between 0.004 and 0.01 produces the correct result of five filaments.

be relevant in the context of any peak-finding algorithm. To quantify the improvement achieved by cross-correlation we compare the $\mathrm{S} / \mathrm{N}$ $\approx 12$ in Figure 5(a), (based on the average peak maximum of 3838 A.U. and the variance of the noise of 317 A.U.) to that observed after application of cross-correlation (Fig. $5(\mathrm{~d}), \mathrm{S} / \mathrm{N} \approx 222$ ). Here we find an improvement of more than an order of magnitude. Note that, in principle, a similar algorithm could also be used to detect the fact that two filaments arrive at the same time. In this case, one would introduce a threshold value distinguishing the intensity from one and two filaments.

Device design. In the following, we discuss how to optimize the signal strength (and thus the $\mathrm{S} / \mathrm{N}$ ratio) and the ability to resolve two close-by filaments, depending on filament length $(l)$ and the detector geometry as described by $w$ and $d$ (see Fig. 1). We first consider a single Au line (Fig. 6(a)). Short filaments $(l<w)$ will 


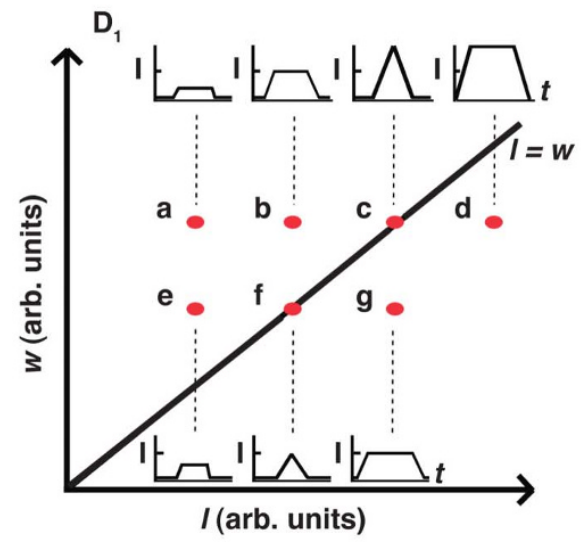

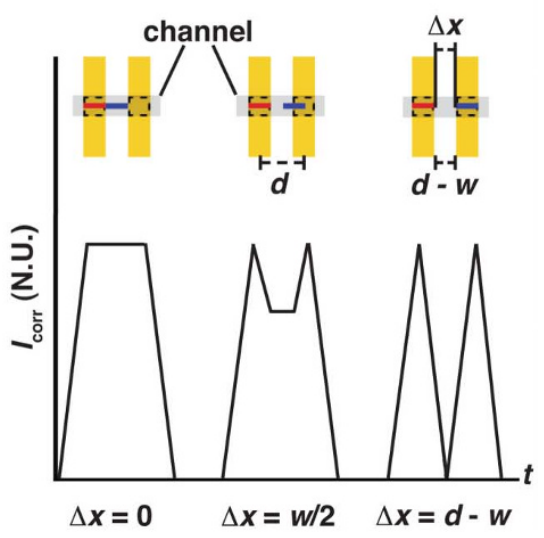

Figure 6 Schematic of signal dependence on filament length $l$ and detector line width $w$ as shown in Figure 1(a). a) Schematic map of expected signal intensity in different locations (labelled $\mathrm{a}-\mathrm{g}$ ) in $\{l, w\}$ parameter space for single filaments. For increasing filament length $l$ (a-b) maximum intensity is obtained for $l=w(\mathrm{c})$. Filaments with $l>w(\mathrm{~d})$ will not yield a higher intensity; they will only have the same intensity for a longer time. Decreasing $w$ decreases signal amplitude (e-g). b) Study of the ability of a pair of detector lines to resolve two filaments travelling with end-to-end distance $\Delta x$ and with $l=w$ for optimal signal strength as a function of $\Delta x$. Two fully resolved peaks are obtained for $\Delta x=d-w$. Inset: schematic of detector geometry and filament end-to-end spacing $\Delta x$.

produce a lower, total FLIC-signal strength, than the optimal value that is achieved for $l \approx w$ (see Fig. 6(a), points a-c). In other words, filaments shorter than the fixed detector width will not yield the maximum possible FLIC-signal, simply due to the fact that they will not fill the detection window at any given time as they pass over it. Filaments with $l>w$ will not produce a stronger signal, but will only broaden the peak (see point $d$ in Fig. 6(a)). This is true, due to the fact that, for filaments longer than the fixed detector width, a maximum FLIC-signal is reached already when the filament has filled the detection window, and therefore the signal cannot increase further as the filament continues to pass over the detection region. Consequently, decreasing $w$ further reduces the signal strength that can be achieved (points e-g). If we therefore assume $l=w$ to be optimal for signal strength, we can set limits on the average filament spacing $\Delta x$ and the line width $w$. When the condition $\Delta x>l+w / 2 \approx(3 / 2) w$ is fulfilled, the signal between two filaments becomes zero, fully resolving the peaks. A somewhat less conservative requirement is $\Delta x>w$. If we then take into consideration that a minimum between two peaks can be fully resolved only if at least three data points are recorded, we obtain an additional condition in the time domain $\Delta t=\Delta x / v>3 f$, where $f$ is the time between frames recorded by the camera, and $v$ is the velocity of the filaments. This second condition can be written as $\Delta x$ $>3 f v$ and is limited by the speed of the camera.

For a two-line detector system (Fig. 6(a)), with the assumption that $l=w$ for optimal signal strength, geometry requires a centre-tocentre line spacing of at least $d>w$ and we assume a reasonable $d$ $=2 w$. The signal $I_{\text {corr }}$ from a pair of detectors (see Fig. 6(b)) will yield two fully separated peaks for two filaments with $\Delta x>d-w$ or $\Delta x>$ $w=l$, using the above assumptions. In this context it is worth noting that, for actin, in standard IVMA procedures, we must require that $l$ $>1 \mu \mathrm{m}$ so that actin filaments maintain motility in the absence of methylcellulose on the surface ${ }^{36}$. These are suitable conditions ${ }^{8,37}$ for relevant nanotechnological applications involving actomyosin. In conclusion, the optimal detector design has pairs of detector lines of width $l \approx w$ and with $\Delta x>w$.

\section{Discussion}

The FLIC method described here is intended for use in devices where large numbers of filaments need to be accurately and automatically recorded in very many different locations of a device, and with minimal data acquisition, for example in a scalable, highly parallel device.
We demonstrated the use of pair-wise detection combined with cross-correlation analysis and thresholding for automatic extraction of filament counts, leading to an increase of the $\mathrm{S} / \mathrm{N}$ ratio by more than an order of magnitude. The use of FLIC to enhance the signal strength in the detector regions enabled an additional increase of $\mathrm{S} / \mathrm{N}$ ratio by a factor of 2 . In total, the techniques described here thus demonstrate an improvement of $\mathrm{S} / \mathrm{N}$ by approximately a factor of 36 compared to standard epifluorescence detection. We believe that these findings can be applied as a platform on which diagnostics, drug discovery and, as discussed here, biocomputation can be carried out.

\section{Methods}

Device design. The overall fabrication process for the device shown in Figure 2 was as follows. The Si substrate is subjected to wet thermal oxidation forming a layer of $\mathrm{SiO}_{2}$ of approximately $845 \mathrm{~nm}$. Lift off resist, LOR 0.7A, (Microchem Corp., Newton, MA, USA) is added by spin coating at 1500 RPM for 30 seconds, with subsequent baking at $180^{\circ} \mathrm{C}$ for 15 minutes on a hotplate. Next, polymethyl methacrylate, PMMA 950A5 (Microchem Corp., Newton, MA, USA) is spin coated on top at 6000 RPM for 60 seconds, followed by baking at $160^{\circ} \mathrm{C}$ for 15 minutes. This double resist layer is exposed to EBL (Raith 150, Dortmund, Germany) for formation of the Au line pattern. After exposure, the PMMA is developed with methyl isobutyl ketone and isopropanol MIBK:IPA (Merck KGaA, Darmstadt, Germany) at a ratio of 1:3 for one minute, followed by rinsing with IPA for 30 seconds. Next, the sample is subject to $\mathrm{O}_{2}$ plasma ashing in a Plasma Preen at $5 \mathrm{mBar}$ for 15 seconds. This plasma ashing step is to remove possible residues of PMMA from the top of the LOR layer before etching. Then, the LOR layer is etched with MF-319: $\mathrm{H}_{2} \mathrm{O}$ (Microposit MF-319 Developer, Rohm and Haas Electronic Materials, Coventry, UK) in the ratio (1:1) for 4 minutes and rinsed with $\mathrm{H}_{2} \mathrm{O}$ for 30 seconds to halt the etching of LOR. $\mathrm{Cr}$ (used as a sticking layer) and $\mathrm{Au}$ are evaporated on the surface to thicknesses of 2 and $40 \mathrm{~nm}$, respectively. The unwanted metal (outside the pattern) and the resists are removed from the surface during a lift off process in Remover 1165 (Microposit Remover 1165, Rohm and Haas Electronic Materials, Coventry, UK) at $80^{\circ} \mathrm{C}$ on a hotplate, with subsequent rinsing in $\mathrm{H}_{2} \mathrm{O}$, leaving behind the desired Au pattern. $\mathrm{HSQ} ; 6 \%$ in MIBK, (XR-1541, Dow Corning, USA) is then spun on top at $1500 \mathrm{RPM}$ for $30 \mathrm{~s}$ and baked at $350^{\circ} \mathrm{C}$ on a hotplate to convert the resist into a $\mathrm{SiO}_{2}$-like layer, see below. A second layer of HSQ; $4 \%$ in MIBK, is then spun on top at $4500 \mathrm{RPM}$ and baked at $350^{\circ} \mathrm{C}$ on a hotplate to convert the resist into a $\mathrm{SiO}_{2}$-like layer, resulting in a total thickness of $d_{2}=240 \mathrm{~nm}$ (see Fig. 3(a)). Nanochannel and loading zone patterns are produced in the same way as the Au line pattern. A cross section of the nanochannel is shown in Figure 2(a).

Fluorescence interference contrast. FLIC is illustrated in Figure 3: fluorophores are excited directly from a source of wavelength $\lambda_{\text {ex }}$, or indirectly by light reflected from a surface (Si or Au in Fig. 3). Light of wavelength $\lambda_{\text {em }}$, emitted from the fluorophores, can travel to the camera directly or indirectly after reflection from the Au surface, after travelling through a semi-transparent oxide layer of $\mathrm{SiO}_{2}$ (thickness $d_{2}$, see Fig. 3a). Differences in the optical pathways of indirect and direct emission pathways give rise to a phase shift causing either constructive or destructive interference $\mathrm{e}^{38-40}$, enhancing 
or quenching the fluorescence respectively. Here, we are interested in constructive interference on top of $\mathrm{Au}$ lines, in order to enhance the fluorescence signal of fluorescently labelled filaments crossing the Au line. Calculation of the fluorescent intensity was performed as $\mathrm{in}^{38}$, according to:

$$
I_{\text {FLIC }}=\sin ^{2}\left(\frac{2 \pi}{\lambda_{e x}}\left(n_{S_{i O_{2}}} d_{2}+n_{w} h_{\text {actin }}\right)\right) \sin ^{2}\left(\frac{2 \pi}{\lambda_{e m}}\left(n_{S_{i O}} d_{2}+n_{w} h_{\text {actin }}\right)\right),
$$

where excitation and emission wavelengths were $540 \mathrm{~nm}$ and $605 \mathrm{~nm}$, respectively, index of refraction for water and $\mathrm{SiO}_{2}$ were $\mathrm{n}_{\mathrm{w}}=1.333$ and $\mathrm{n}_{\mathrm{SiO} 2}=1.46$, and for a fluorophore height of $h_{\text {actin }}=40 \mathrm{~nm}$, i.e. the height that actin is held above the surface ${ }^{41}$. This fluorescence intensity as a function of $d_{2}$ indicates the first fluorescence maxima at approximately $d_{2}=50 \mathrm{~nm}$ and $d_{2}=240 \mathrm{~nm}$ above Au, see Figure 3(b). Due to instabilities of very thin HSQ layers, the larger $d_{2}=240 \mathrm{~nm}$ was used. For the thickness of the $\mathrm{SiO}_{2}$ layer on top of the Si substrate $\left(d_{1}\right.$ in Fig. 3(a)), we use $845 \mathrm{~nm}$, effectively eliminating any FLIC effect above the Si. If desired, one can additionally use the destructive FLIC effect to eliminate fluorescence off the Au lines, by tuning $d_{1}$ and $d_{2}$.

HSQ application. We used the negative-tone resist hydrogen silsesquioxane (HSQ) to coat the $\mathrm{Au}$ lines. This resist is transformed into a $\mathrm{SiO}_{2}-$ like layer ${ }^{42}$, when baked at high temperatures or exposed to EBL. HSQ has been used in a variety of applications ${ }^{43,44}$ and is a suitable substitute for forming $\mathrm{SiO}_{2}$ films by direct deposition $^{45}$. HSQ can also, as shown here, support actomyosin motility. Here we have chosen to use HSQ as $\mathrm{a} \mathrm{SiO}_{2}$ layer due to its ease of use. Other techniques such as atomic layer deposition (ALD) have advantages and drawbacks compared to using HSQ. Using ALD one can apply a highly uniform $\mathrm{SiO}_{2}$ layer, however, due to the deposition method, a repeating sandwich layer of $\mathrm{Al}_{2} \mathrm{O}_{3}$ and $\mathrm{SiO}_{2}$ must be used to increase thickness ${ }^{46}$. This may affect the FLIC signals and has therefore not been considered.

Surface treatments for actomyosin motility. After formation of nanochannels, plasma treatment was used to ensure that the top resist layer of PMMA was sufficiently hydrophilic and therefore unable to support motility ${ }^{5}$. As a next step, silanization using chemical vapour phase deposition (CVD) of $98 \%$ (GC) trimethylchlorosilane, TMCS (Sigma-Aldrich Sweden AB, Stockholm, Sweden) was performed to promote motility on the floor of the loading zones and nanochannels, namely the exposed SiOx substrate ${ }^{28}$. Caution: TMCS is highly flammable and reacts violently with water! Wetting of the surface was done to reduce the possibility of air bubbles forming in the channels ${ }^{4}$.

Fluorescence microscopy. Rhodamine Phalloidin ( $\mathrm{RhPh}$ ) labelled filaments were observed using an inverted fluorescence microscope (Nikon, Eclipse TE300) equipped with a 100x oil immersion objective (Nikon, NA 1.4) and a Tetra methyl Rhodamine Iso-Thiocyanate (TRITC) filter set (Ex 540/25, DM 565, BA 605/25). Images were recorded with an EMCCD camera (Hamamatsu C9100) and analyzed with Image J (Rasband, W.S ImageJ, U.S. National Institutes of Health, Bethesda, Maryland, USA, http://imagej.nih.gov/ij/, 1997-2012). Actin filament sliding velocities were calculated using a previously described Matlab algorithm ${ }^{32}$. This was done by manually following the tip of actin filaments moving in the nanostructured channel. All in vitro motility assays (IVMAs) were performed with the actomyosin system as described previously ${ }^{37,41}$. In brief, proteins were diluted in buffer A (10 mM MOPS, $1 \mathrm{mM} \mathrm{MgCl}_{2}, 1 \mathrm{mM}$ DTT, $0.1 \mathrm{mM}$ EGTA, $50 \mathrm{mM} \mathrm{KCl}, \mathrm{pH}$ 7.4). Flow cells were then incubated as follows: (i) $120 \mu \mathrm{g} / \mathrm{ml} \mathrm{HMM} \mathrm{for} 4$ minutes (ii) $1 \mathrm{mg} / \mathrm{ml}$ BSA for 1 minute (iii) $1 \mu \mathrm{M}$ block actin (short and unlabeled actin filaments) in Buffer A with $1 \mathrm{mM}$ ATP for 2 minutes (iv) rinsed with a 40 (buffer A with final concentrations of $10 \mathrm{mM}$ DTT and $25 \mathrm{mM} \mathrm{KCl}, 1 \mathrm{mM}$ ATP, $2.5 \mathrm{mM}$ creatine phosphate, 56 units $/ \mathrm{ml}$ creatine kinase) (v) rinsed with Buffer A (vi) $100 \mathrm{nM} \mathrm{RhPh}$ labelled actin filaments for 1 minute (vii) rinsed with Buffer A (viii) a60 (a40 with final concentrations of $45 \mathrm{mM}$ $\mathrm{KCl}, 3 \mathrm{mg} / \mathrm{ml}$ glucose, 20 units $/ \mathrm{ml}$ glucose oxidase, 460 units $/ \mathrm{ml}$ catalase. The motility assays were performed at $26^{\circ} \mathrm{C}$.

1. Kron, S. J. \& Spudich, J. A. Fluorescent actin filaments move on myosin fixed to a glass surface. Proc. Natl. Acad. Sci. 83, 6272-6276 (1986).

2. Kron, S. J., Toyoshima, Y. Y., Uyeda, T. Q. \& Spudich, J. A. Assays for actin sliding movement over myosin-coated surfaces. Methods Enzymol. 196, 399-416 (1991).

3. Howard, J., Hudspeth, A. J. \& Vale, R. D. Movement of microtubules by single kinesin molecules. Nature 342, 154-158 (1989).

4. Bunk, R. et al. Guiding motor-propelled molecules with nanoscale precision through silanized bi-channel structures. Nanotechnology 16, 710-717 (2005).

5. Bunk, R. et al. Actomyosin motility on nanostructured surfaces. Biochem. Biophys. Res. Commun. 301, 783-788 (2003).

6. Hess, H., Clemmens, J., Qin, D., Howard, J. \& Vogel, V. Light-controlled molecular shuttles made from motor proteins carrying cargo on engineered surfaces. Nano Lett 1, 235-239 (2001).

7. Hiratsuka, Y., Tada, T., Oiwa, K., Kanayama, T. \& Uyeda, T. Q. Controlling the direction of kinesin-driven microtubule movements along microlithographic tracks. Biophys. J. 81, 1555-1561 (2001).

8. Sundberg, M. et al. Actin filament guidance on a chip: toward high-throughput assays and lab-on-a-chip applications. Langmuir 22, 7286-7295 (2006).

9. Mansson, A. et al. In vitro sliding of actin filaments labelled with single quantum dots. Biochem. Biophys. Res. Commun. 314, 529-534 (2004).
10. Ramachandran, S., Ernst, K. H., Bachand, G. D., Vogel, V. \& Hess, H. Selective loading of kinesin-powered molecular shuttles with protein cargo and its application to biosensing. Small 2, 330-334 (2006).

11. Kumar, S. et al. Antibodies Covalently Immobilized on Actin Filaments for Fast Myosin Driven Analyte Transport. PLoS ONE 7, e46298 (2012).

12. Paguirigan, A. L. \& Beebe, D. J. Microfluidics meet cell biology: bridging the gap by validation and application of microscale techniques for cell biological assays. BioEssays 30, 811-821 (2008).

13. Squires, T. M. \& Quake, S. R. Microfluidics: fluid physics at the nanoliter scale. Rev. Mod. Phys. 77, 977-1026 (2005).

14. Whitesides, G. M. The origins and the future of microfluidics. Nature 442, 368-373 (2006).

15. Wang, J. Cargo-towing synthetic nanomachines: towards active transport in microchip devices. Lab Chip 12, 1944-1950 (2012).

16. van den Heuvel, M. G. L. \& Dekker, C. Motor proteins at work for nanotechnology. Science 317, 333-336 (2007)

17. Takatsuki, H. et al. Transport of single cells using an actin bundle-myosin bionanomotor transport system. Nanotechnology 22, 245101 (2011).

18. Patolsky, F., Weizmann, Y. \& Willner, I. Actin-based metallic nanowires as bionanotransporters. Nat. Mater. 3, 692-695 (2004).

19. Agarwal, A. \& Hess, H. Biomolecular motors at the intersection of nanotechnology and polymer science. Prog. Polym. Sci. 35, 252-277 (2010).

20. Fischer, T., Agarwal, A. \& Hess, H. A smart dust biosensor powered by kinesin motors. Nat. Nanotech. 4, 162-166 (2009).

21. van den Heuvel, M. G., de Graaff, M. P. \& Dekker, C. Molecular sorting by electrical steering of microtubules in kinesin-coated channels. Science 312, 910-914 (2006).

22. Meyhofer, E. \& Howard, J. The force generated by a single kinesin molecule against an elastic load. Proc. Natl. Acad. Sci. U. S. A. 92, 574-578 (1995).

23. Nicolau Jr., D. V., Burrage, K. \& Nicolau, D. V. Computing with motile bio-agents. Proc. SPIE-Int. Soc. Opt. Eng. 6416 (2007).

24. Nicolau, D. V. et al. Molecular motors-based micro- and nano-biocomputation devices. Microelectron Eng 83, 1582-1588 (2006).

25. Nicolau Jr., D. V. \& Nicolau, D. V. Biocomputation schemes based on the directed and directional movements of motile biological objects. Proc. SPIE 5651, 134-143 (2005).

26. Adleman, L. M. Molecular computation of solutions to combinatorial problems. Science 266, 1021-1024 (1994).

27. Rozenberg, G. \& Salomaa, A. Vol. 1644 Lecture Notes in Computer Science (eds Jirí Wiedermann, Peter van Emde Boas, \& Mogens Nielsen) 106-118 (Springer Berlin/Heidelberg, 1999).

28. Sundberg, M. et al. Silanized surfaces for in vitro studies of actomyosin function and nanotechnology applications. Anal. Biochem. 323, 127-138 (2003).

29. Ruhnow, F., Zwicker, D. \& Diez, S. Tracking single particles and elongated filaments with nanometer precision. Biophys. J. 100, 2820-2828 (2011)

30. Ernest, N. C., Wall, R. A., Jed, C. M. \& George, H. Speckled microtubules improve tracking in motor-protein gliding assays. Phys. Bio. 4, 10 (2007).

31. Hamelink, W., Zegers, J. G., Treijtel, B. W. \& Blangé, T. Path reconstruction as a tool for actin filament speed determination in the in vitro motility assay. Anal. Biochem. 273, 12-19 (1999).

32. Mansson, A. \& Tagerud, S. Multivariate statistics in analysis of data from the in vitro motility assay. Anal. Biochem. 314, 281-293 (2003)

33. Haustein, E. \& Schwille, P. Fluorescence correlation spectroscopy: novel variations of an established technique. Annu. Rev. Biophys. Biomol. Struct. 36, 151-169 (2007).

34. Du, P., Kibbe, W. A. \& Lin, S. M. Improved peak detection in mass spectrum by incorporating continuous wavelet transform-based pattern matching. Bioinformatics 22, 2059-2065 (2006).

35. Pyenta, P. S., Holowka, D. \& Baird, B. Cross-correlation analysis of inner-leafletanchored green fluorescent protein co-redistributed with IgE receptors and outer leaflet lipid raft components. Biophys. J. 80, 2120-2132 (2001).

36. Toyoshima, Y. Y., Kron, S. J. \& Spudich, J. A. The myosin step size: measurement of the unit displacement per ATP hydrolyzed in an in vitro assay. Proc. Natl. Acad. Sci. 87, 7130-7134 (1990).

37. Sundberg, M. et al. Selective spatial localization of actomyosin motor function by chemical surface patterning. Langmuir 22, 7302-7312 (2006).

38. Parthasarathy, R. \& Groves, J. T. Optical techniques for imaging membrane topography. Cell Biochem. Biophys. 41, 391-414 (2004).

39. Lambacher, A. \& Fromherz, P. Luminescence of dye molecules on oxidized silicon and fluorescence interference contrast microscopy of biomembranes. J Opt Soc Am B 19, 1435-1453 (2002).

40. Kerssemakers, J., Howard, J., Hess, H. \& Diez, S. The distance that kinesin-1 holds its cargo from the microtubule surface measured by fluorescence interference contrast microscopy. Proc. Natl. Acad. Sci. U. S. A. 103, 15812-15817 (2006).

41. Persson, M. et al. Heavy meromyosin molecules extending more than $50 \mathrm{~nm}$ above adsorbing electronegative surfaces. Langmuir 26, 9927-9936 (2010).

42. Yang, C.-C. \& Chen, W.-C. The structures and properties of hydrogen silsesquioxane (HSQ) films produced by thermal curing. J. Mater. Chem. 12, 1138-1141 (2002).

43. Namatsu, H. et al. Three-dimensional siloxane resist for the formation of nanopatterns with minimum linewidth fluctuations. J. Vac. Sci. Technol., B 16, 69-76 (1998). 
44. Egard, M. et al. High transconductance self-aligned gate-last surface channel In0.53Ga0.47As MOSFET. Proc. IEEE IEDM, 13, 12.11-13.12.14 (2011).

45. Arkles, B. et al. Staged development of modified silicon dioxide films. J. Sol-Gel Sci. Technol. 8, 465-469 (1997).

46. Hausmann, D., Becker, J., Shenglong, W. \& Gordon, R. G. Rapid vapor deposition of highly conformal silica nanolaminates. Science 298, 402 (2002).

\section{Acknowledgements}

The work leading to this invention has received funding from the European Union Seventh Framework Programme (FP7/2007-2011) under grant agreement $n^{\circ} 228971$ (Molecular Motors-based Nano Devices -MONAD), the Swedish Research Council under grant $\mathrm{n}$ 621-2010-5146, nmC@LU, the Knut and Alice Wallenberg Foundation, and the Carl Trygger's foundation.

\section{Author contributions}

M.L. planned the study, performed the experiments, analyzed the results and wrote the paper. LtS performed the experiments and contributed to the analysis. A.M. and H.L. conceived and initiated the study, contributed to the analysis and edited the paper.

\section{Additional information}

Competing financial interests: The authors declare no competing financial interests. License: This work is licensed under a Creative Commons

Attribution-NonCommercial-NoDerivs 3.0 Unported License. To view a copy of this license, visit http://creativecommons.org/licenses/by-nc-nd/3.0/

How to cite this article: Lard, M., Siethoff, L.t., Månsson, A. \& Linke, H. Tracking Actomyosin at Fluorescence Check Points. Sci. Rep. 3, 1092; DOI:10.1038/srep01092 (2013) 